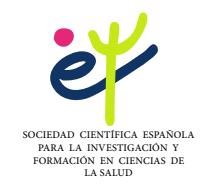

European Journal of Child Development,

Education and Psychopathology

2021, Vol. 9, No 1 (Págs. 1-14)

Eur. J. Develop. Educa. Psychop. ISSN 2340-924X https://revistas.uautonoma.cl/index.php/ejpad doi: $10.32457 /$ ejpad.v9i1.1403

\title{
Reflexiones sobre el Absentismo en las aulas universitarias
}

\section{Reflections about the Absenteeism in University classrooms}

\author{
Esther Raya Diez $^{1 *}$ (D) y Fermin Navaridas Nalda ${ }^{2}$ \\ ${ }^{1}$ Facultad de Ciencias Jurídicas y Sociales, Universidad de la Rioja, España; ${ }^{2}$ Facultad de Letras \\ y de la Educación, Universidad de la Rioja, España
}

\section{RESUMEN}

En este trabajo se define la asistencia significativa como el grado en que los estudiantes perciben que las clases impartidas por sus profesores posibilitan su desarrollo personal y profesional futuro, contribuyendo a la adquisición de competencias básicas y específicas definidas en el perfil de egreso de su titulación. Sobre la base este planteamiento, el problema objeto de estudio gira en torno a la preocupación docente respecto al incremento del absentismo universitario en las clases expositivas o magistrales. Para responder a dicho problema se proponen los siguientes objetivos: a) describir el comportamiento de asistencia a las clases de grupo grande en titulaciones de grado; b) identificar los factores asociados a comportamientos de absentismo en el alumnado universitario; y c) comprender los aspectos valorados por parte de los estudiantes para una asistencia significativa. El diseño metodológico para recabar información adopta un enfoque mixto, mediante la combinación de técnicas de investigación cualitativas y cuantitativas. El universo de estudio lo han conformado las universidades del campus Iberus. Se ha realizado un grupo focal con docentes universitarios y se ha pasado un cuestionario estructurado a estudiantes de diferentes grados y cursos. Entre las conclusiones obtenidas, cabe destacar que el problema del absentismo es un fenómeno complejo que debe ser analizado desde enfoques multifactoriales. En el nuevo escenario de la Educación Superior, resulta conveniente plantearse qué se espera de la presencialidad en el aula universitaria para que la asistencia sea realmente significativa.

Palabras clave: Absentismo universitario, percepción, asistencia significativa, Educación Superior

* Para correspondencia: Dra. Esther Raya Diez, Facultad de Ciencias Jurídicas y Sociales, Edificio Quintiliano, Calle Cigüeńa, 60, 26004 Logrońo (La Rioja). España. Correo: esther.raya@unirioja.es 


\begin{abstract}
In this work, significant attendance is defined as the extent to which students perceive that the taken classes promote their personal and professional development and contribute to the acquisition of basic and specific skills defined in their university degree program. Based on this approach, the problem under study focuses on the teachers' concern regarding the increase in university student absenteeism that is particularly observed in lectures. To answer this problem, the following objectives were proposed: a) to describe the behavior of attending large group classes in undergraduate degrees; $b$ ) to identify the factors associated with absenteeism behaviors in university students; and c) to understand which are the most valued aspects by students concerning significant attendance. The methodological design for collecting information adopted a mixed approach, combining qualitative and quantitative research techniques. The statistical universe of this study includes the four universities that make up the Campus Iberus. A focus group was established with university teachers and then a structured questionnaire was passed to their students, which belong to different courses and grades. Among the conclusions of this study, it should be noted that the problem of absenteeism is a complex phenomenon that must be analyzed from multifactorial approaches. In the new scenario of Higher Education, it should be kept in mind what is expected of physical presence in the university classroom so that the attendance is really significant.
\end{abstract}

Keywords: University student absenteeism, perception, significant attendance, Higher Education

\title{
1. INTRODUCCIÓN
}

En términos generales, algunos indicadores relativos a las tasas de absentismo y de éxito académico de los estudiantes universitarios (Ministerio de Ciencia, Innovación y Universidades, 2019; Unión Europea, 2019) parecen indicar la necesidad de repensar los procesos de enseńanza y aprendizaje que tienen lugar durante la actividad docente, así como la necesidad de dar solución a un problema de asistencia que no resulta del todo significativa para un número relativamente importante de estudiantes univesitarios. En este trabajo, como punto de partida, consideramos que la asistencia significativa representa el grado en que los estudiantes perciben que las clases impartidas por sus profesores posibilitan su desarrollo personal y profesional futuro, contribuyendo a la adquisición de competencias básicas y específicas definidas en el perfil de egreso de su titulación, y haciendo que disfruten con la actividad académica, se entusiasmen con las tareas emprendidas y encuentren utilidad al conocimiento desarrollado durante las mismas. Para algunos autores (Pintrich y Schunk, 2002; Alonso, 2005), cuando esto ocurre se dice que los estudiantes presentan 
una motivación intrínseca, atribuyéndole a las clases presenciales en el aula el significado señalado y con efectos muy positivos en la calidad de los procesos de aprendizaje resultantes.

Con este mismo propósito, la creación del Espacio Europeo de Educación Superior (EEES) (Declaración de Bolonia, 1999) supuso un cambio metodológico en el modelo tradicional de enseñanza universitaria (De Miguel, 2006; López, 2011) y sobre el papel de la función docente en la sociedad tecnológicamente avanzada. En el Comunicado de Londres de 2007 los ministros de educación plantean propuestas metodológicas para promover el aprendizaje eficaz de los estudiantes universitarios en un mundo globalizado (Comunicado de Londres, 2007). Se adoptaron los ECTS como nuevo sistema de medición de la actividad de los estudiantes, y con ello, se ha generado una nueva forma de organización de la actividad docente, poniendo en el centro del proceso el protagonismo del estudiante y promoviendo un enfoque basado en competencias (De Miguel, 2006; López, 2011). Con ello se ha tratado de dar cabida al desarrollo de actividades más prácticas y significativas, vinculadas a lo profesional y no solo mediante estrategias docentes basadas en las clases expositivas y magistrales.

En el caso concreto de las universidad española, es habitual diferenciar la actividad docente entre grupos grandes (GG) y grupos reducidos (GR). Los primeros, con un carácter más teórico y los segundos de formación práctica, orientados al desarrollo y transferencia del conocimiento adquirido previamente en contextos significativos de aprendizaje, asegurando de esta forma el desempeño competencial por parte de los estudiantes.

Sin embargo el absentismo en la enseñanza universitaria entra en conflicto con las premisas básicas que se defienden en el EEES en materia de autonomía, compromiso y responsabilidad del estudiante con el propio aprendizaje (Álvarez y López, 2011). Se comienza a observar un incremento del absentismo, más acusado en las clases de GG, donde la presencialidad no es percibida como obligatoria. Este comportamiento ha sido objeto de estudio en la ámbito de la educación superior (Jiménez y Rodríguez, 2010; Crespo, Palomo y Méndez, 2012; Rao, Valleswary, Nayas et al., 2016; Magobolo y Dube, 2019).

El absentismo ha sido definido como "la acción consciente y premeditada de ausentarse del medio físico de la clase universitaria, el aula, condicionada por una serie de factores, y que conduce a la búsqueda de alternativas en el uso del tiempo" (Moral, Navarro y García, 2010). Esta ausencia a clase intencional puede tener consecuencias importantes tanto en el rendimiento académico de los estudiantes que adoptan con frecuencia dicho comportamiento (Dashputra, Kulkarni, Chari, et al., 2015), como también en la adquisición de competencias que son consideradas básicas para su desarrollo personal y el desempeño eficaz de las responsabilidades inherentes al ejercicio de su profesión futura (Navaridas, Jiménez y Fernández, 2016).

El fenómeno del absentismo de los estudiantes universitarios parece atribuirse a diferentes factores, encontrando más de un significado. Asi, por ejemplo, algunos trabajos de 
investigación (Jiménez y Rodríguez, 2010) señalan la factores relacionados con el contexto docente, entre los que se puede mencionar el sistema metodológico y el tipo de evaluación; aspectos socioacadémicos característicos de los estudiantes (por ejemplo, motivos de acceso a la titulación cursada, objetivos personales a cuya consecución conceden más importancia). Otros autores (Thekedam y Kottaram, 2015) destacan de forma complementaria la influencia de factores relacionados con el valor o la utilidad percibida a corto y a largo plazo de las clases impartidas por sus profesores (por ejemplo, la adquisición de aprendizajes que posibiliten resolver problemas reales en un contexto profesional cercano, el acceso a puestos específicos de trabajo, el acceso a estudios superiores o, simplemente, conseguir calificaciones positivas para el objetivo perseguido). Otras investigaciones (Crespo, Palomo y Méndez, 2012; Mahmoud, 2017) atribuyen especial importancia a factores relacionados con problemas personales (por ejemplo, enfermedades físicas, cuidar de un familiar, simultaneidad de estudios o responsabilidades laborales). Todo ello plantea la necesidad de contemplar un modelo que nos permita analizar los factores críticos que debemos tener en cuenta para entender este comportamiento por parte de los estudiantes en el contexto académico, y de esta forma saber sobre qué aspectos tratar de influir y cómo hacerlo.

En este marco, encontramos diversos modelos de estudio que permiten profundizar sobre los factores identificados como posibles determinantes de la actitud y el comportamiento final de los estudiantes frente a las clases pesenciales (Davis, 1985; Davis, Bagozzi \& Warshaw, 1989; Venkatesh \& Davis, 2000), dando lugar a esquemas de análisis muy interesantes con base a los que diseñar contextos de aprendizaje que promuevan y faciliten la asistencia significativa de los estudiantes. Muchos de ellos, en su diseño inicial se basan en la Teoría de la Acción Razonada (Davis, 1989) que plantea que las conductas de las personas dependen de su sistema interno de creencias sobre las consecuencias de su comportamiento y la influencia social en dicha conducta (norma subjetiva). Aunque la gran mayoría de estas teorías han ido evolucionando y se ha centrado principalmente en desarrollar la capacidad de comprender y predecir la intención de comportamiento en contextos diversos relacionados con la aceptación tecnológica, consideramos interesante partir del modelo propuesto por Davis (1989) como un esquema de estudio flexible y útil para explicar los comportamientos de absentismo de los estudiantes en las aulas universitarias (Fig. 1). Del mismo modo, pensamos también que este modelo puede ser utilizado como un esquema de gran interés para la reflexión docente sobre el grado de significatividad de las clases presenciales y sus consecuencias académicas en los estudiantes objeto de estudio.

A partir de este modelo de análisis, los objetivos de este trabajo consisten en: a) describir el comportamiento de asistencia a las clases de grupo grande en titulaciones de grado; b) identificar los factores asociados a comportamientos de absentismo en el alumnado universitario; y b) comprender los aspectos valorados por parte de los estudiantes para una asistencia significativa. 


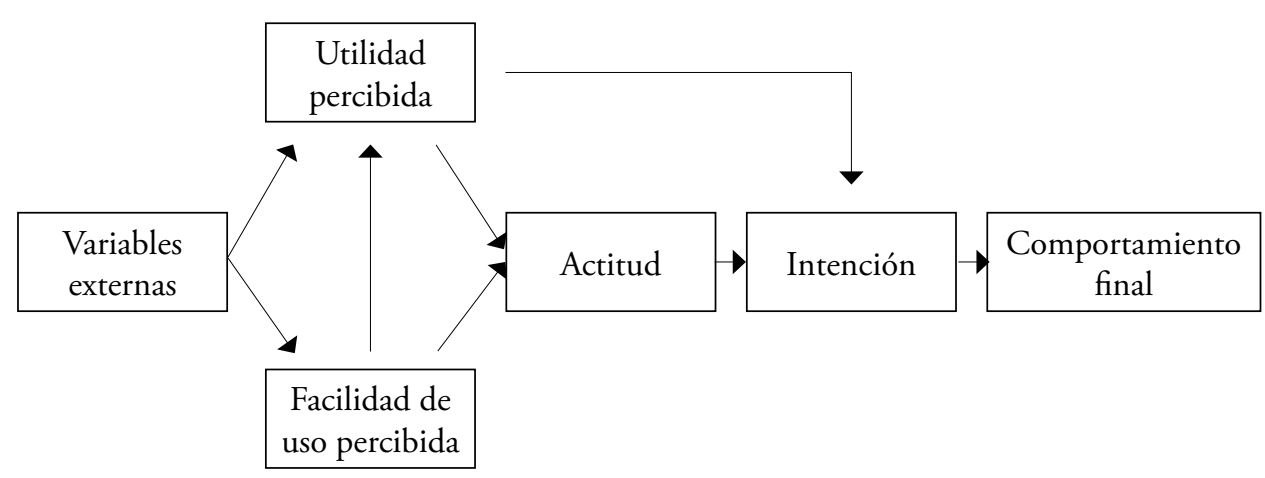

Fuente: Factores incluidos en el Modelo de estudio desarrollado por Davis (1989).

\section{METODOLOGÍA}

El estudio se ha realizado a través de un diseño metodológico mixto, con combinación de técnicas cualitativas y cuantitativas. El universo de estudio lo han conformado las universidades del campus Iberus* (Universidad de Zaragoza, Universidad Pública de Navarra, Universidad de Lleida y Universidad de La Rioja), junto a la Universidad del País Vasco/Euskal Herriko Unibertsitatea.

Se ha realizado un grupo focal compuesto por diez profesores con características heterogéneas en cuanto a edad, género, formación académica y área de conocimiento, con actividad docente en diferentes titulaciones de grado y másteres universitario, tales como Derecho, Adminsitración y Dirección de Empresas, Maestro en Educación Primaria, Maestro en Educación Infantil, Ingeniería y Trabajo Social, entre otras.

En su mayoría cuentan con amplia experiencia docente. El grupo focal se centró en dos cuestiones relativas a la asistencia de los estudiantes a las asignaturas presenciales y los factores que desde su punto de vista influyen en la asistencia a las clases de teoría de GG.

Por otro lado, se ha recogido información directa de los estudiantes de los grados seńalado a través de un cuestionario semiestructurado, compuesto por preguntas tipo lickert sobre creencias en torno al proceso de enseñanza aprendizaje; preguntas cerradas sobre comportamiento relativo a la asistencia presencial; y, finalmente variables sociodemográficas. Se ha obtenido una muestra intencional compuesta por 319 estudiantes. La recogida de

* $\quad$ El Campus Iberus es una asociación estratégica de las universidades públicas de las comunidades autónomas de Aragón, La Rioja, Comunidad Foral de Navarra y la de la provincia de Lleida en Cataluña. 
información se realizó en la segunda quincena del mes de marzo, en el inicio del estado de alarma. En la tabla 1 se presentan las características sociodemográficas del alumnado participante.

\section{Tabla 1:}

Perfil del alumnado.

\begin{tabular}{lrr}
\hline Características & Abs. & $\%$ \\
\hline Mujer & 237 & 74,29 \\
Hombre & 78 & 24,45 \\
Sin datos & 4 & 1,25 \\
& 319 & 100,00 \\
Universidad de La Rioja & 156 & 48,90 \\
Universidad Pública de Navarra & 17 & 5,33 \\
Universidad de Zaragoza & 73 & 22,88 \\
Universidad de Lleida & 55 & 17,24 \\
Universidad del País Vasco/ Euskal Herriko & 18 & 5,64 \\
Unibertsitatea & 319 & 100,00 \\
& 140 & 43,89 \\
Grado en Trabajo Social & 86 & 26,96 \\
Grado en Educación & 12 & 3,76 \\
Grado en Administración y Dirección de empresas & 11 & 3,45 \\
Grado en Derecho & 39 & 12,23 \\
Grado en Ingeniería & 19 & 5,96 \\
Otros Grados & 6 & 1,88 \\
Otros estudios de master & 619 & 1,88 \\
Dobles titulaciones & & 100,00 \\
\hline
\end{tabular}

Fuente: Elaboración propia.

\section{RESULTADOS}

Se presentan a continuación el avance de resultados, destacando en primer lugar, la valoración del profesorado y en segundo lugar, las respuestas de los estudiantes. 


\subsection{Grupo focal: la percepción docente}

La percepción docente sobre la asistencia del alumnado a las clases presenciales destaca aspectos absentistas, aunque se observan diferencias dentro del alumnado. Este comportamiento es descrito en los siguientes términos:

tendencia a reducir la asistencia a medida que avanza el semestre (P2)

El alumnado que asiste regularmente es colaborador, participativo y regular. Hay otro alumnado que es poco constante, aparece de vez en cuando en clase. (P5)

Tiende a venir las dos terceras partes del curso de manera continua (P6)

Los estudiantes asisten con regularidad a las sesiones programadas. Aunque hay un grupo que por circunstancias personales está desvinculado. (P7)

Asisten regularmente hasta las tres o cuatro semanas previas al examen (P8)

Las clases teóricas ven reducida la presencia de alumnos conforme pasa el cuatrimestre (P11)

En el discurso docente se pueden identificar un conjunto de factores como aspectos explicativos de los comportamientos de asistencia a las clases presenciales de los estudiantes universitarios.

En un primer nivel los discursos aluden a factores de microcontexto. En el mismo se incluyen, por un lado, aspectos relativos a las características propias de los y las estudiantes, tales como su percepción de la utilidad de las clases; la influencia del grupo de iguales; la percepción de la capacidad para aprobar sin asistir a clases. En este nivel el discurso docente describe los comportamientos de los estudiantes en los siguientes términos:

Creo que los estudiantes consideran que la asistencia a las sesiones teóricas no resultan imprescindibles y que pueden sustituirse por apuntes obtenidos de otros estudiantes (P1)

Y por otro lado, aspectos relacionados con la función docente, como son la metodología de enseñanza aprendizaje y el sistema de evaluación.

Realizan en todo momento cómputos de "coste de oportunidad" y "coste-beneficio" para saber cómo comportarse en una asignatura. Deciden que comportamiento tener bajo la funcionalidad del aprobado: ¿a cuántas clases puedo faltar? ¿a qué sesiones es imprescindible que asista?" (P4).

Influye la puntuación por evaluación continua (P7)

Cuando la asignatura se entiende importante en su formación o la han elegido porque les motiva, la actitud y el comportamiento son claramente buenos. Cuando no es asi, hay desinterés, absentismo, lenguaje no verbal inapropiado"(P9).

Las metodologias empleadas (activa y significativa). El rol docente como facilitador del aprendizaje (12)

que la asistencia se cuente a la hora de las notas (P14) 
Un segundo nivel de factores se refieren a aspectos de nivel intermedio o mesocontexto. En ellos se destacan las condiciones del contexto de enseńanza, con elementos como la estructura de los planes de estudio y la organización de la docencia.

creo que lo que debemos repensar es el número de horas presenciales que estamos exigiendo a nuestros alumnos en cada asignatura. Una menor presencialidad exigiría una forma diferente de abordar la enseñanza por parte del profesor, que debería tutorizar y acompañar al alumno en su proceso de aprendizaje. Creo asimismo que la rigidez de los horarios —iguales para todas las asignaturas - dificulta acomodarse a las especificidades de cada materia.

También aparecen los aspectos relativos a la coordinación docente:

"el hecho de señalar semanalmente una actividad en grupo reducido que, en ocasiones, requiere la realización por parte del alumno de ciertas tareas previas, les lleva a optar por destinar buena parte de su jornada a la realización de esas otras tareas". (P1).

"Saturación de trabajos." (P4).

"la realización de exámenes eliminatorios de otras asignaturas fuera de convocatoria reducen considerablemente la asistencia" (P7).

Asimismo cabría identificar un tercer nivel o macrocontexto que estaría relacionado con aspectos de la sociedad de la información, como son el acceso a Internet y la disminución de la capacidad de atención ante la hiperconectividad. La asistencia al aula responde más a una constumbre o tradición, aunque no va vinculada a una participación activa en la clase que se manifiesta en conductas disruptivas o de déficit de atención:

La actitud es, en general, pasiva, resultando muy difícil conseguir interactuar con los alumnos. (P2).

Su comportamiento es dispar, proclive a hablar y a distraerse en clase (P3)

Decepcionante. Si estableces asistencia obligatoria los alumnos vienen sin motivación, hay que llamarles la atención (uso de móviles, corrillos de conversaciones, etc) y te bombardean con justificantes para la no-asistencia. Si es asistencia libre hay una deserción amplia (la justifican porque es "la única" en la que no se controla asistencia y necesitan un rato de descanso (P4)

"Deficit de atención"( P8)

Comportamiento infantil, tendencia al murmullo, falta de atención y actitudes prepotentes en algunos casos. Escaso nivel conceptual y déficit de atención (P9).

La asistencia es habitual y mayoritaria. Sin embargo, parece obedecer más a un tradición en la cultura del estudiante que a un interés efectivo. La triada clase-apuntes-examen parece prevalecer en la mentalidad del estudiante. El comportamiento es, en su conjunto, pasivo (P10) 
La actitud de los alumnos tanto en Teoría (grupo grande) como en grupo reducido es de escucha pasiva, sin participación, aún cuando es requerida de forma directa, les cuesta dar una opinión, argumentar o contraargumentar, contar una experiencia, poner un ejemplo, compartir el análisis de una lectura que han trabajado en casa o en clase...la verdad es me cuesta motivarlos para la acción. No obstante, en todos los grupos hay dos o tres personas más participativas y el resto del grupo delega en estas personas su participación. Por otro lado, a veces se da la situación en la que el propio grupo censura a aquellos alumnos más participativos con risas, murmullos y miradas cómplices entre ellos y reprobatorias hacia el participante. (P12)

\subsection{Cuestionario: Actitudes y comportamiento de los estudiantes universitarios respecto a la asistencia}

A partir de las respuestas obtenidas del cuestionario, el primer aspecto a destacar es que los estudiantes que en mayor medida han contestado al mismo son los que presentan un mayor nivel de asistencia a clase. El 75,2\% señalan que acuden de forma regular, a más del $75 \%$ de las clases.

Las respuestas de los estudiantes respecto al nivel de asistencia general del alumnado a las clases presenciales de grupo grande, se presentan divididas, entre quienes señalan respuestas de asistencia superiores a la mitad del alumnado matriculado (mucha y bastante asistencia, $44 \%$ de respuestas); y quienes opinan que la asistencia es inferior a la mitad del alumnado matriculado $43 \%$. Entre ambas opiniones un $13 \%$ responden que la asistencia es variable de unas asignaturas a otras.

\section{Gráfico 1:}

Asistencia en general del alumnado a las clases presenciales de $G G$, según la opinión de los estudiantes.

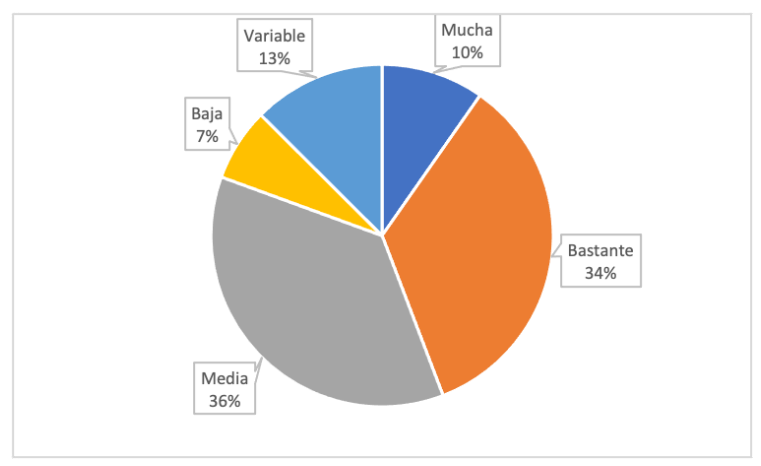

Elaboración propia. 
La proporción de estudiantes que señalan que la asistencia es muy variable de unas asignaturas a otras, es un indicio de la incidencia de los factores metodológicos en la toma de decisión respecto al compartamiento absentista.

La valoración de los estudiantes sobre la relación entre asistencia regular y aprendizaje, muestra los siguientes resultados:

\section{Gráfico 2:}

Valoración del item "el aprendizaje es mayor en las clases donde asisto regularmente".

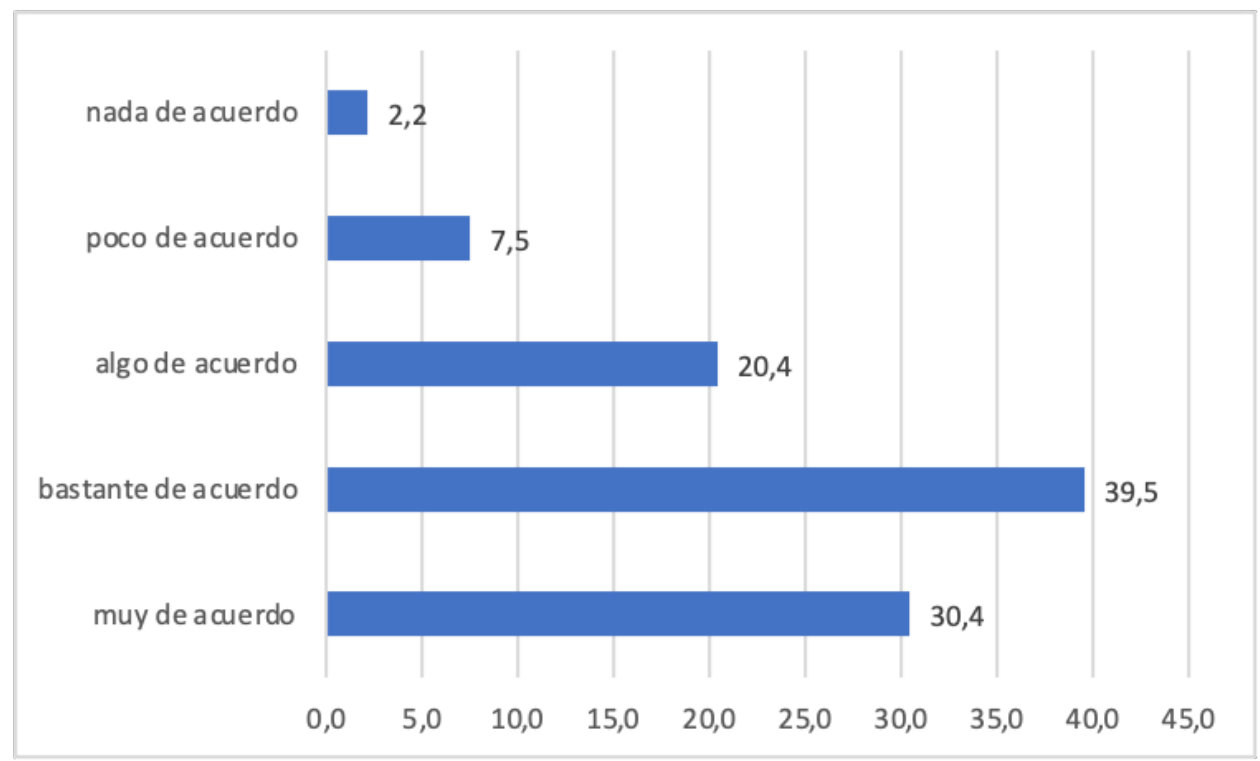

\section{Elaboración propia.}

Si bien la opinión mayoritaria (bastante y muy de acuerdo) considera que el aprendizaje es mayor cuando se asiste regularmente. Una cuarta parte de los estudiantes presentan dudas respecto a la afirmación.

Asimismo, de forma mayoritaria responden que asistiría en mayor medida si el profesorado utilizara una metodología activa. 


\section{Gráfico 3:}

Valoración del item "Asistiria más si el profesorado utilizara una metodología activa".

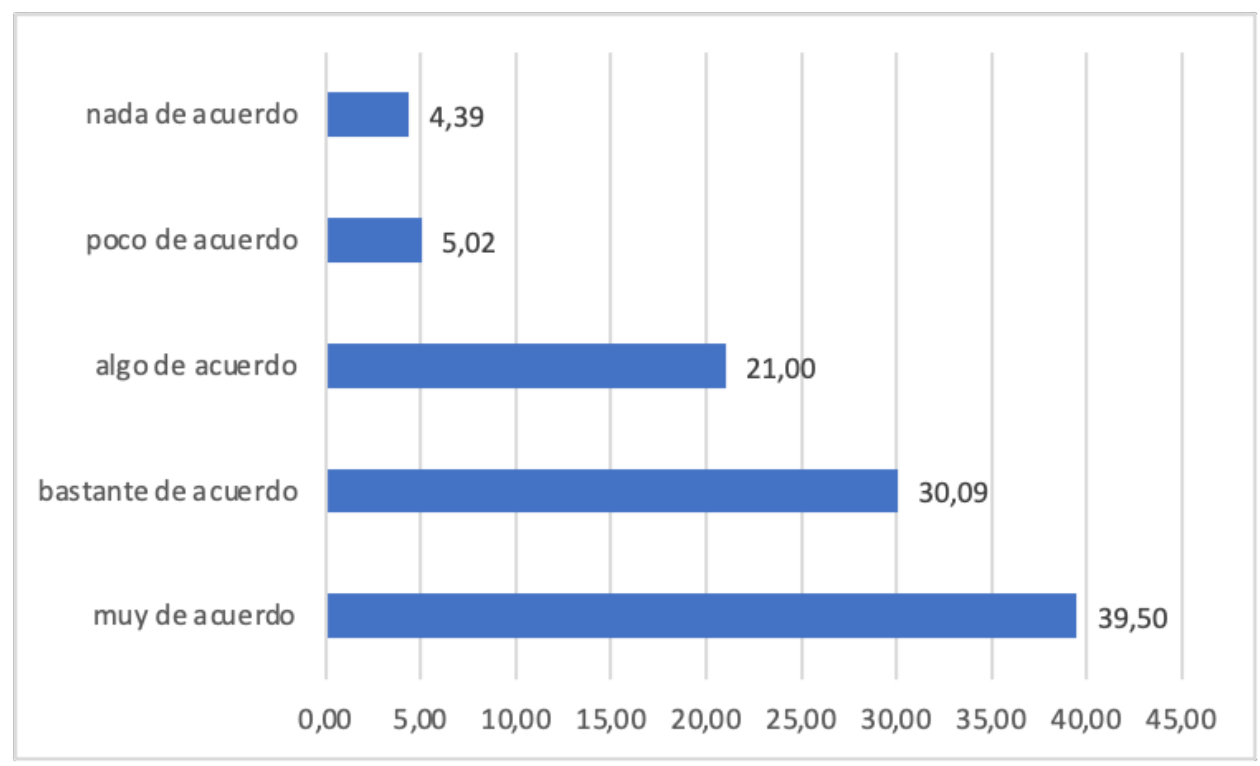

Elaboración propia.

Como se observa en el gráfico, los resultados presentan una figura en escalera, con un 69\% de respuestas bastante de acuerdo o muy de acuerdo con la información.

\section{CONCLUSIONES}

La universidad en cuanto institución de Educación Superior tiene un papel relevante e indiscutible en la formación especializada y de alto nivel. El Espacio Europeo de Educación Superior ha supuesto un cambio en los modelos organizativos y pedagógicos en el aula universitaria en general. Además, las sociedades postglobalizadas (Saldańa, 2010), emergentes con el inicio del siglo XXI, mediante la extensión de las tecnologías de la información y la comunicación representan un nuevo contexto en el cual se desempeńa la actividad docente en todos los niveles educativos, incluido el universitario. Más allá de los aspectos geopolíticos y económicos, y aunque sus características todavía se presentan difusas, desde el punto de vista sociocultural parece apuntar hacia procesos de individualización donde el yoismo "nos hace pensar que todo el entorno debe ser favorable a nuestros intereses" (Benedetto, 2020). 
En las aulas universitarias el absentismo estudiantil comienza a ser preocupante. La bibliografía consultada tiende a destacar aspectos relacionados con el contexto docente, relativos a la metodología y evaluación de la asignatura; aspectos relacionados con las características socioacadémicas de los estudiantes, como es la motivación por los estudios; también otros aspectos relacionados con la utilidad percibida de ir a clase; y finalmente, cuestiones personales.

Estos factores han sido apuntados por el profesorado consultado en este estudio, quienes destacan por un lado, factores de microcontexto relativos a los procesos de interacción dentro del aula; la utilidad y el interés percibido del estudiante respecto a la asistencia. Entre los factores mesocontexto aluden a cuestiones como la planificación y la organización de la docencia, junto con la coordinación docente. En tercer lugar, entre los factores macrocontexto seńalan, principalmente, la falta de atención en clase y dispersión. En este sentido, se debe recordar la influencia de la hiperconectividad en estudiantes nativos digitales, habituados a recibir material audiovisual y contenidos multimedia.

Por su parte, los estudiantes, consultados en este trabajo, reconocen mayoritariamente que se obtienen mejores resultados de aprendizaje cuando se asiste regularmente a clase. Asimismo, consideran que la asistencia aumentaría si se utilizasen metodologías activas que les diera protagonismo y participación en la construcción del conocimiento.

En síntesis, cabe destacar que el comportamiento respecto a la asistencia o falta de la misma por parte de los estudiantes dificilmente se puede reducir a un único factor. Debe ser analizado desde enfoques multifactoriales y multicausales, que permitan garantizar un aprendizaje de calidad. En este nuevo contexto de sociedad postglobalizada y, atravesada por una pandemia mundial, parece más necesario que nunca plantearse qué se espera de la presencialidad en el aula universitaria, para que la asistencia sea significativa, tal como ha sido definida en este trabajo. Entendiendo por ello, la presencia en el aula mediante la escucha activa y la participación directa y atenta a las actividades y dinámicas de la sesión que derive un un aprendizaje significativo. La pandemia de la Covid-19 ha marcado la actividad docente de la actualidad universitaria; sin embargo el reto va más allá de la cuestión modalidad presencial o modalidad online, sino que requiere una revisión en profundidad de los modos de aprender y enseñar en la sociedad postglobal.

\section{Agradecimientos}

Este trabajo forma parte del proyecto de innovación docente financiado por la Universidad de La Rioja en la convocatoria de 2019 titulado Asistencia Significativa: Retos de la enseñanza presencial (37/2019). Nuestro agradecimiento a los docentes que forman parte del equipo de investigación. 


\section{REFERENCIAS}

Alonso Tapia, J. (2005). La motivación para el aprendizaje: la perspectiva de los alumnos. En Ministerio de Educación y Ciencia. La orientación escolar en centros educativos. (pp.209-242).Madrid: MEC.

Álvarez, P. R. y López, D. (2011). El absentismo en la enseñanza universitaria : un obstáculo para la participación y el trabajo autónomo del alumnado. Bordón, 63(3), 43-56

Benedetto, Humberto (2020) Un mundo de contradicciones secundarias: La Post Globalización? En Parlamento del Mercosur, disponible en: https://tinyurl.com/y4dth52u

Comunicado de Londres, Hacia el Espacio Europeo de Educación Superior: respondiendo a los retos de un mundo glo- balizado, Londres 17-18 de mayo de 2007. En: https:// tinyurl.com/y63gleua

Crespo, N. M.T. Palomo \& M. Méndez, "El efecto del absentismo universitario en el expediente académico y en la percepción de sus causas" EDUCADE. Revista de Educación en Contabilidad, Finanzas y Administración de Empresas, 3, pp. 47-65, 2012

Dashputra, A.; Kulkarni, M.; Chari, S. y Date, A. (2015). Medical students' absenteeism in class: Reasons and remedies, Journal of Educational Research and Studies, 3(1), 2429. Davis F. (1989): "Perceived usefulness, perceived ease of use and user acceptance of information technology”, MIS Quarterly, 13(3), pp. 319-340.

Davis, F. D. (1985): A Technology Acceptance Model for Empirically Testing New End-User Information Systems: Theory and Results. Massachusetts Institute of Technology.

Davis, F. D., Bagozzi, R. P., \& Warshaw, P. R. (1989): User Acceptance of Computer Technology: A Comparison of Two Theoretical Models. Management Science, 35(8), 982-1003.

De Miguel, M. 2006; (dir.) Modalidades de enseñanza centradas en el desarrollo de competencias. Orientaciones para promover el cambio metodológico en el Espacio Europeo de Educación Superior Oviedo: Ediciones Universidad de Oviedo, 2006.

Declaración de Bolonia, Espacio Europeo de Enseñanza Superior. Declaración conjunta de los ministros europeos de enseńanza reunidos en Bolonia el 19 de junio de 1999. Retrieved from: https://tinyurl.com/y5vz2g5n

Del Moral, L., L. Navarro y M. García “Absentismo estudiantil en la Escuela Universitaria de Estudios Empresarial de la Universidad de Sevilla" in El absentismo en las aulas universitarias. El caso de la escuela universitaria de estudios empresariales de la Universidad de Sevilla (J.L. Jiménez y A. Rodríguez eds.) 15-58, Sevilla: Grupo Editorial Universitario, 2010

Jiménez, J.L. \& A. Rodríguez, El absentismo en las aulas universitarias. El caso de la escuela universitaria de estudios empresariales de la Universidad de Sevilla. Sevilla: Grupo Editorial Universitario, 2010 
López, J.L. "Un giro copernicano en la enseñanza universitaria: formación por competencias”, Revista de Educación, 356, pp. 279-301., 2011

Magobolo, G.N. \& B.D. Dube, "Factors influencing high absenteeism rate of student nurses in clinical areas at a nursing college in the Lejweleputswa Distric." Curationis, 42(1), a1985, https://doi.org/10.4102/curationis.v42i1.1985, 2019

Mahmoud , S.S. "An analytical study into absenteeism in Saudi higher education institutions: Causes and possible solutions." IOSR Journal of Research \& Method in Education, 7(5), 20-31. 2017

Ministerio de Ciencia, Innovación y Universidades (2019). Datos y cifras del Sistema universitario español: Publicación 2018-2019. Madrid: Secretaría General Técnica del Ministerio de Ciencia, Innovación y Universidades. Recuperado de: https://tinyurl. com/yyqajrd4

Navaridas, F.; Jiménez, M.A. \& Fernández, R.(2016). El aprendizaje de competencias en la Universidad: expectativas predictivas y niveles de confirmación de los estudiantes. Revista Española de Pedagogía, 74 (64), pp. 337-356.

Pintrich, P. R., \& Schunk, D. H. (2002). Motivation in Education: Theory, Research and Applications (2nd ed.). Upper Saddle River, NJ: Prentice Hall.

Rao, B.T., K. Valleswary, K., M.S.D.P. Nayak \& N.L. Rao, "Reasons for Absenteeism among the Undergraduate Medical Students Attending for Theory Classes in Rajiv Gandhi Institute of Medical Sciences (RIMS) Ongole, Prakasam District of Andhra Pradesh: A Self Review". IOSR Journal of Research \& Method in Education (IOSRJRME) 6(4), 11-19, 2016;

Saldaña, E. (2010) Matices de la educación en la condición postglobal, Matices. Revista de Postgrado. Universidad Nacional Autónoma de Nuevo México, Vol. 5, No13, pp.261-280

Thekedam, J.S. \& Kottaram, J.S., 2015, Strategic intervention techniques to reduce chronic absenteeism and truancy among students, International Journal of Management \& Business Studies 5(2), 1-14.

Unión Europea (2019). Monitor de la Educación y la Formación de 2019. España. Luxemburgo: Oficina de Publicaciones de la Unión Europea. http://sepie.es/doc/ comunicacion/publicaciones/2020/et-monitor-report-2019-spain_es.pdf

Venkatesh, V. \& F.D. Davis, F. D. "A theoretical extension of the technology acceptance model: Four longitudinal field studies2. Management Science, 46(2), 186-204, 2000

Recibido: 30 de octubre de 2020 Aceptado: 15 de noviembre de 2020 